\title{
Оптимизация условий анализа рисперидона и продуктов его разложения методом ВЭЖХ
}

\author{
Голубицкий Г.Б., Владимирова Е.В., Островский К.П., Гельперина С.Э. \\ ООО «Технология лекарств», Химки \\ Поступила в редакцию 16.08.2017 г.
}

\begin{abstract}
Оптимизировано разделение рисперидона и продуктов его разложения в обращенно-фазовой системе. Сделано предположение о химической природе основного продукта разложения рисперидона в присутствии пероксида водорода. Показаны возможности использования подвижной фазы, содержащей перхлорат аммония, для улучшения разделения пиков в исследуемой системе. Впервые изучено влияние ацетонитрила и диметилсульфоксида на скорость разложения рисперидона в присутствии пероксида водорода в водно-органическом растворе. Органические растворители ускоряют деструкцию, что объясняется механизмом реакции. Влияние диметилсульфоксида значительно слабее, что связано с его более высокой диэлектрической проницаемостью по сравнению с ацетонитрилом, а также со способностью реагировать с пероксидом водорода.

Ключевые слова. Рисперидон, рисперидон-цис-N-оксид, окислительное разложение, обращенно-фазовая система, влияние ацетонитрила, влияние диметилсульфоксида, летучие компоненты подвижной фазы, прозрачность в коротковолновой области УФ-спектра.
\end{abstract}

\section{Optimization of HPLC analysis conditions for risperidone and its decomposition products}

\author{
Golubitsky G.B., Vladimirova E.V., Ostrovskiy K.P., Gelperina S.E.
}

LTD «Drugs Technology», Khimki

\begin{abstract}
The purpose of this study is optimization of HPLC analysis conditions for risperidone and its decomposition products for the development of quantitative analysis for prolonged medicinal forms, and application of the mobile phase based on volatile, transparent in the short wavelength ultraviolet region of the spectrum components to approach the way to unify the chromatography analysis.

We optimized conditions for separation of risperidone and its decomposition products in reversedphase system. We compared the retention time received for degradation products at different $\mathrm{pH}$ of the mobile phase with the data from literature, and the assumption is made about the chemical nature of the main product of decomposition of risperidone in the presence of hydrogen peroxide. We showed the possibilities of use of mobile phase containing water, acetonitrile, perchloric acid and ammonia to optimise the separation of peaks of the test system. We determined kinetic parameters for risperidone decomposition in the presence of hydrogen peroxide. We have studied for the first time the effect of acetonitrile and dimethyl sulfoxide on the rate of risperidone decomposition in the presence of hydrogen peroxide in the aqueous-organic solution. We showed that organic solvents accelerate the degradation due to the changed dielectric constant of the medium. The effect of dimethyl sulfoxide is much weaker, because of its higher dielectric constant compared to acetonitrile, and ability to react with hydrogen peroxide.

Keywords. Risperidone, risperidone-cis-N-oxide, oxidative decomposition, reversed-phase system, the influence of acetonitrile, the influence of dimethyl sulfoxide, the volatile components of the mobile phase, transparency in the shortwave region of the UV spectrum.
\end{abstract}




\section{Введение}

Рисперидон (РС) - высокоэффективное лекарственное средство, применяемое в психиатрии. Это органическое основание с $\mathrm{pK}$ a $=8.76$ [1].

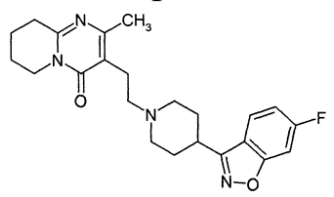

Согласно литературным данным, подходы к анализу препаратов РС методом ВЭЖХ чрезвычайно разнообразны. Так, для количественного определения РС и примесей в аналогичных препаратах (таблетки РС и таблетки РС жевательные) методики фармакопеи США (USP 36) значительно различаются - градиентный и изократический режимы соответственно при одинаковом качественном составе подвижной фазы (ПФ) [2, стр. 5065-5068]. Чаще всего рН буферных составляющих ПФ близок к 7 [3], иногда используют буферные растворы с $\mathrm{pH} 3 \div 5$ [2,4]. Поскольку РС и его примеси - вещества ионной природы, это различие приводит к различным результатам разделения. Оптимизация разделения производится не всегда. Например, на колонке $4.6 \times 250$ мм с предколонкой 4.6×12.5 мм при скорости потока ПФ $1.0 \mathrm{~cm}^{3} /$ мин для одного из основных продуктов разложения РС $t_{R}=2.6$ мин, т.е. удерживание отсутствует [5]. Изучено влияние кислоты, щелочи и окислителя на стабильность РС в водных растворах [3]. Публикации о важной для пробоподготовки роли органического растворителя нами не найдены.

Различие методик анализа аналогичных препаратов усложняет и удорожает работу аналитических лабораторий. Поэтому теоретически интересен и практически перспективен подход к разработке хроматографических методик, предусматривающий их унификацию по составу ПФ и режиму элюирования. Барам и соавт. для решения этой задачи в качестве ПФ использовали смесь ацетонитрила с $0.2 \mathrm{M}$ раствором перхлората лития с добавлением $0.1 \mathrm{M}$ раствора хлорной кислоты до $\mathrm{pH} 2.8$. Анализ проводили в градиентном режиме с изменением концентрации ацетонитрила в ПФ от 5 до 100 об. \%. Достоинство данного состава ПФ - высокая растворимость перхлората лития в воде и ацетонитриле, низкое поглощение в коротковолновой области УФ-спектра [6].

Цель настоящей работы - оптимизация условий анализа РС и продуктов его разложения методом ВЭЖХ для разработки методики количественного анализа пролонгированной лекарственной формы и развитие подхода к унификации хроматографических методик с использованием ПФ на основе летучих компонентов, имеющих высокую растворимость в воде и низкое поглощение в коротковолновой области УФ-спектра.

\section{Эксперимент}

Использовали жидкостный хроматограф Prominence LC-20 с автоматическим дозатором SIL-20AC и диодно-матричным детектором SPD-M20A (Shimadzu), а также pH-метр Seven Compact S20 с комбинированным стеклянным электродом (Mettler Toledo).

Удерживание РС при различных рН ПФ изучали на колонке размером 4.6×250 мм с защитной предколонкой 20×4.6 мм, заполненных сорбентом XBridge Shield RP18 с размером частиц 5 мкм (Waters), для остальных экспериментов использовали колонку размером 4.6×100 мм с защитной предколонкой $20 \times 4.6$ мм с аналогичным сорбентом с размером частиц 3.5 мкм. 
Для приготовления ПФ использовали смеси ацетонитрила с водными растворами $(0.5$ об. \%) хлорной или трифторуксусной кислот. $\mathrm{pH}$ растворов кислот доводили до необходимого значения водным раствором аммиака с потенциометрическим контролем. Расход ПФ составил $1.0 \mathrm{~cm}^{3} /$ мин, объем проб 20 мкл, температура колонок $40^{\circ} \mathrm{C}$, образцов $25^{\circ} \mathrm{C}$, длина волны детектирования 237 нм, соответствующая коротковолновому максимуму поглощения РС.

Использовали сверхчистую воду из установки Advantage A10 (Millipore), peактивы фирмы PanReac - ацетонитрил UHPLC Supergradient, трифторуксусную кислоту, гидроксид натрия и фирмы Sigma Aldrich - водный раствор аммиака (25 масс. $\%)$, хлорную кислоту (70 масс. \%), пероксид водорода (35 масс. \%) (все реактивы, кроме ацетонитрила - reagent grade), РС фармакопейного качества (содержание основного вещества $99.6 \%$ согласно паспорту лекарственного средства, производство - Китай).

\section{Обсуждение результатов}

Зависимость удерживания РС от $\mathrm{pH}$ и состава ПФ (3:7) $\mathrm{CH}_{3} \mathrm{CN}-(0.5$ об. \% кислота $+\mathrm{NH}_{3}$ до необходимого $\mathrm{pH}$ ) исследовали в диапазоне $\mathrm{pH} 1.8 \div 9.6$. Время выхода пика неудерживаемого вещества (2.90 мин) оценивали по пику растворителя (смесь (3:7) ацетонитрил - вода). Результаты представлены в табл. 1 и на рис. 1.

Таблица 1. Зависимость удерживания и формы пика рисперидона от $\mathrm{pH}$ и состава подвижной фазы

\begin{tabular}{|c|c|c|c|c|c|c|c|}
\hline Состав ПФ & $\mathrm{pH}$ & 1.8 & 2.9 & 4.3 & 6.4 & 8.1 & 9.6 \\
\hline \multirow{5}{*}{$\begin{array}{c}\text { (3:7) } \mathrm{CH}_{3} \mathrm{CN}- \\
0.5 \text { об. } \% \mathrm{HClO}_{4}+\mathrm{NH}_{3}\end{array}$} & $t_{R}$. мин & 10.80 & 12.00 & 12.20 & 12.40 & 15.40 & 24.60 \\
\hline & $k_{l}$ & 2.72 & 3.14 & 3.21 & 3.28 & 4.31 & 7.48 \\
\hline & $\ln k_{1}$ & 1.00 & 1.14 & 1.17 & 1.19 & 1.46 & 2.01 \\
\hline & $A_{s}$ & 1.280 & 1.220 & 1.230 & 1.210 & 1.220 & 1.200 \\
\hline & $N$ & 10660 & 10670 & 10700 & 10950 & 11510 & 10780 \\
\hline \multirow{5}{*}{$\begin{array}{c}\text { (3:7) } \mathrm{CH}_{3} \mathrm{CN}- \\
0.5 \text { об. \% } \\
\mathrm{CF}_{3} \mathrm{COOH}+\mathrm{NH}_{3}\end{array}$} & $t_{R}$. Мин & 6.40 & 8.70 & 9.70 & 9.90 & 13.90 & 24.70 \\
\hline & $k_{2}$ & 1.21 & 2.00 & 2.34 & 2.41 & 3.79 & 7.52 \\
\hline & $\ln k_{2}$ & 0.19 & 0.69 & 0.85 & 0.88 & 1.33 & 2.02 \\
\hline & $A_{s}$ & 1.630 & 1.370 & 1.250 & 1.260 & 1.180 & 1.180 \\
\hline & $N$ & 7970 & 10630 & 11630 & 11640 & 14230 & 17460 \\
\hline \multicolumn{2}{|l|}{$\ln k_{1} / \ln k_{2}$} & 5.33 & 1.65 & 1.37 & 1.35 & 1.10 & 1.00 \\
\hline
\end{tabular}

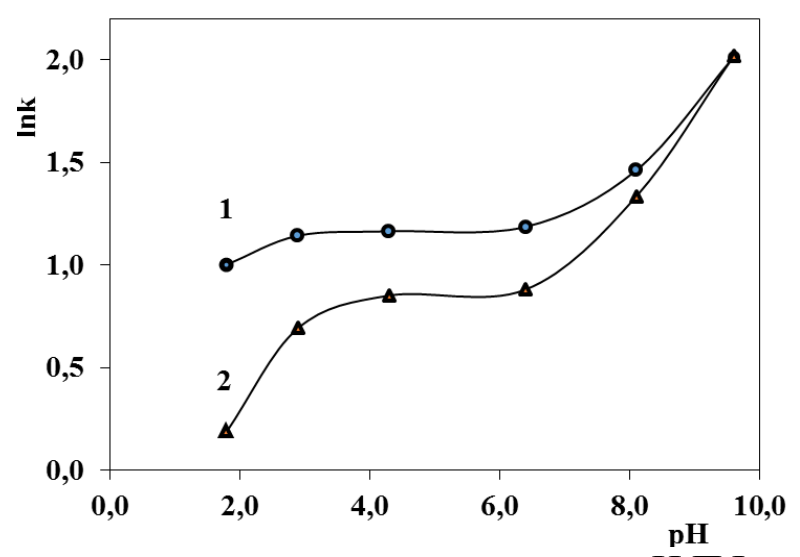

Рис. 1. Зависимость удерживания рисперидона от $\mathrm{pH}$ ПФ на основе кислот:

1 - хлорной; 2 - трифторуксусной. 
Возрастание удерживания РС в диапазоне $\mathrm{pH}$ 8.1-9.6 соответствует депротонизации молекулы в соответствии с $\mathrm{pK}_{\mathrm{a}}=8.76$. При использовании $\mathrm{HClO}_{4}$ удерживание РС возрастает быстрее, чем в случае $\mathrm{CF}_{3} \mathrm{COOH}$. Это объясняется хаотропным эффектом, который проявляется в десольватации молекул протонированного основания при образовании ими гидрофобных ионных пар с анионами $\mathrm{ClO}_{4}^{-}$[7-9]. C pocтом $\mathrm{pH}$ ПФ различие удерживания РС в присутствии разных кислот снижается и при значениях $\mathrm{pH}>\mathrm{pK}_{\mathrm{a}}$ исчезает $\left(\ln k_{1} / \ln k_{2} \rightarrow 1\right)$, поскольку при этих условиях РС переходит в молекулярную форму, не образующую пар с анионами. В присутствии $\mathrm{HClO}_{4}$ коэффициент асимметрии $A_{S}$ пика $\mathrm{PC}$ во всем изученном диапазоне $\mathrm{pH}$ изменяется незначительно. Способность $\mathrm{CF}_{3} \mathrm{COOH}$ к образованию ионных пар ниже по сравнению с $\mathrm{HClO}_{4}$ [9], поэтому выше роль перехода РС в молекулярную форму. В данном случае, по мере роста рН ПФ значение $A_{S}$ пика РС снижается, а эффективность колонки $N$ по пику РС растет, поскольку нейтральная молекула РС слабее взаимодействует с остаточными силанольными группами сорбента. Рекомендуемый для анализа диапазон $\mathrm{pH}$ буферной составляющей ПФ 6.7-7.3: при более низких $\mathrm{pH}$ ухудшается разделение пиков продуктов разложения РС, при более высоких - исчезает возможность использования хаотропного эффекта для оптимизации разделения.

Зависимость удерживания РС от концентрации перхлорат-иона в ПФ (23:77) $\mathrm{CH}_{3} \mathrm{CN}-\left(\mathrm{HClO}_{4}+\mathrm{NH}_{3}\right.$ до $\left.\mathrm{pH} 7.0\right)$ изучена для подтверждения роли хаотропного эффекта в исследуемой системе. Время выхода пика неудерживаемого вещества (1.36 мин) оценивали по пику растворителя (смесь (3:7) ацетонитрил - вода). Полученные результаты представлены в табл. 2.

Таблица 2. Зависимость эффективности колонки, удерживания и формы пика рисперидона от концентрации перхлорат-иона в буферной составляющей ПФ с $\mathrm{pH} 7.0$ $(\mathrm{P}=0.95 ; \mathrm{n}=3)$

\begin{tabular}{|c|c|c|c|c|}
\hline $\begin{array}{c}\text { Концентрация } \\
\mathrm{ClO}_{4}^{-} \\
\mathrm{M}\end{array}$ & $\begin{array}{c}\text { Время удержи- } \\
\text { вания РС } t_{R}, \\
\text { мин }\end{array}$ & $\operatorname{lnk}$ & $\begin{array}{c}\text { Эффективность ко- } \\
\text { лонки по пику РС, } N\end{array}$ & $\begin{array}{c}\text { Коэффициент } \\
\text { асимметрии пика } \\
\text { РС, } A_{S}\end{array}$ \\
\hline 0.000 & $11.9 \pm 0.1$ & $1.73 \pm 0.01$ & $290 \pm 30$ & $1.080 \pm 0.05$ \\
\hline 0.009 & $12.5 \pm 0.1$ & $1.78 \pm 0.01$ & $8530 \pm 160$ & $1.270 \pm 0.06$ \\
\hline 0.018 & $13.3 \pm 0.1$ & $1.82 \pm 0.01$ & $8920 \pm 210$ & $1.310 \pm 0.07$ \\
\hline 0.026 & $14.1 \pm 0.1$ & $1.87 \pm 0.01$ & $9340 \pm 240$ & $1.280 \pm 0.06$ \\
\hline 0.044 & $14.8 \pm 0.1$ & $1.91 \pm 0.01$ & $9610 \pm 250$ & $1.250 \pm 0.05$ \\
\hline 0.061 & $15.2 \pm 0.1$ & $1.93 \pm 0.01$ & $9920 \pm 270$ & $1.220 \pm 0.05$ \\
\hline 0.088 & $15.6 \pm 0.1$ & $1.95 \pm 0.01$ & $9990 \pm 270$ & $1.220 \pm 0.05$ \\
\hline
\end{tabular}

При отсутствии $\mathrm{ClO}_{4}^{-}$в ПФ пик РС симметричен, однако эффективность колонки $N$ по пику РС неприемлемо низка. При вводе $0.009 \mathrm{M} \mathrm{HClO}_{4}$ эффективность резко возрастает и при дальнейшем росте $\left[\mathrm{ClO}_{4}{ }^{-}\right]$изменяется незначительно. Удерживание РС в изученном диапазоне концентрации монотонно растет, а зависимость коэффициента асимметрии $A_{S}$ от $\left[\mathrm{ClO}_{4}^{-}\right]$имеет максимум. Согласно этим данным, для работы рекомендуется диапазон $\left[\mathrm{ClO}_{4}{ }^{-}\right]$0.05-0.09M (0.3-0.5 об. \%), обеспечивающий оптимальные значения эффективности колонки по пику РС и фактора асимметрии пика РС.

Режим элюирования РС и продуктов его разложения. Разделение РС и продуктов его разложения возможно как в изократическом, так и в градиентном режимах (рис. 2 а, б). Градиентный режим более предпочтителен, поскольку позволяет повысить пиковую емкость хроматограммы, улучшить селективность разделения и чувствительность по сравнению с изократическим вариантом. При переходе от изократики к градиенту коэффициент асимметрии пика РС практически не изменяется 
$\left(A_{S} \approx 1.20\right)$, однако ширина пика на половине высоты снижается почти в два раза (с 0.40 до 0.22 мин). Разрешение между пиками основных продуктов разложения при переходе от изократики к градиенту возрастает от 7.6 до 10.5. Рекомендуемая скорость изменения концентрации $\mathrm{CH}_{3} \mathrm{CN}$ в ПФ - от 20 до 30 об. \% в течение 20 мин. Увеличение крутизны градиента ускоряет анализ, но ухудшает разделение пиков продуктов разложения. Кроме того, при более крутом градиенте имеет место так называемый «эффект памяти» [10], когда на хроматограммах чистого растворителя наблюдается пик РС. Хроматограмма, представленная на рис. 2,б, может быть использована для характеристики пригодности используемой хроматографической системы для анализа РС и продуктов его разложения.
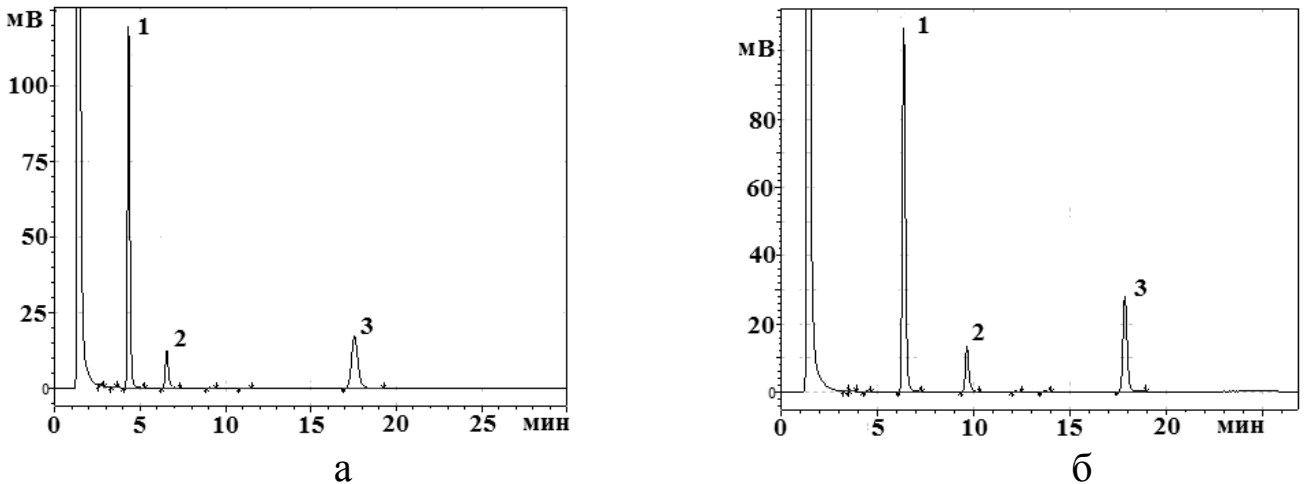

Рис. 2. Хроматограммы раствора РС (57.0 мкг/см 3$)$ в смеси (3:7) $\mathrm{CH}_{3} \mathrm{CN}-3 \%$ раствор $\mathrm{H}_{2} \mathrm{O}_{2}$ через 4.0 ч (а) и 4.5 ч (б) после приготовления раствора. ПФ (23:77) $\mathrm{CH}_{3} \mathrm{CN}-0.5$ об. \% $\mathrm{HClO}_{4} \mathrm{pH} 7.3\left(\mathrm{NH}_{3}\right)$ (а) и линейный градиент от 20 до 30 об. \% $\mathrm{CH}_{3} \mathrm{CN}$ за 20 мин - 0.5 об. \% $\mathrm{HClO}_{4} \mathrm{pH} 7.3\left(\mathrm{NH}_{3}\right)$ (б). 1 - рисперидон-цис-N-оксид; 2 - неидентифицированный продукт разложения РС; 3 - РС.

Влияние концентрации ацетонитрила и диметилсульфоксида в испытуемом растворе на скорость окислительного разложения РС. Исследование стабильности РС при воздействии кислоты, щелочи, температуры и окислителя подтвердило литературные данные [3] о максимальной чувствительности этого вещества к окислению. Установлено, что добавление $\mathrm{CH}_{3} \mathrm{CN}$ в раствор РС ускоряет его окислительное разложение (рис. 3). Линейность зависимостей натурального логарифма площади пика РС от времени (коэффициент корреляции $r>0.99$ ) отражает псевдопервый порядок реакции разложения по концентрации РС, для которого выполняется соотношение:

$$
\ln c_{\tau}=\ln c_{0}-k \tau
$$

где $c_{\tau}$ и $c_{0}$ - концентрации РС в момент времени $\tau$ и начальный момент реакции соответственно, М; $k$ - константа скорости реакции разложения РС, мин ${ }^{-1}$. Методом наименьших квадратов для линейной регрессии рассчитаны значения константы скорости реакции разложения $\mathrm{PC}$ в присутствии $\mathrm{H}_{2} \mathrm{O}_{2}$ при разном содержании $\mathrm{CH}_{3} \mathrm{CN}$ в растворе, численно равные коэффициентам наклона линейных зависимостей. Полученные результаты представлены в табл. 3. Учитывая, что РС практически не растворим в воде [1], в анализируемые растворы необходимо добавлять, как минимум, 5 об. \% $\mathrm{CH}_{3} \mathrm{CN}$.

Для ДМСО получены качественно аналогичные результаты: при росте его содержания в растворе разложение РС ускоряется. Однако, влияние этого ратворителя значительно слабее, чем $\mathrm{CH}_{3} \mathrm{CN}$ : при добавлении в раствор от 5 до 50 об. \% ДМСО площадь пика РС в течение 80 мин после приготовления раствора не изменяется, а площадь пика основного продукта разложения возрастает до 0.3\% (5 об. \% ДМСО) и до $0.6 \%$ (50 об. \% ДМСО). 


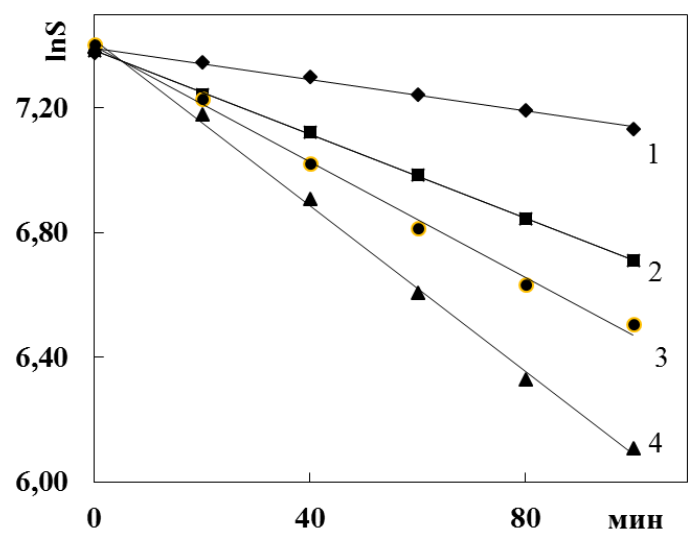

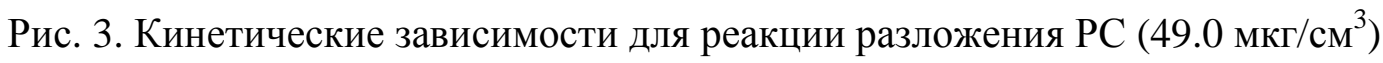
при концентрации $\mathrm{CH}_{3} \mathrm{CN}$ в испытуемом растворе, об. \%: 1-5; 2-20; 3-30; 4-50. Содержание $\mathrm{H}_{2} \mathrm{O}_{2}$ в растворах 3 масс. \%. По оси ординат - натуральные логарифмы площадей пика РС; исходные площади в мкВ×с

Таблица 3. Зависимость константы скорости разложения рисперидона от содержания ацетонитрила в $3 \%$ растворе пероксида водорода

\begin{tabular}{|c|c|}
\hline Концентрация $\mathrm{CH}_{3} \mathrm{CN}$, об. $\%$ & $\begin{array}{c}\text { Константа скорости разложения РС. } \text { мин }^{-1} \\
(\mathrm{P}=0.95 ; \mathrm{n}=6)\end{array}$ \\
\hline 5.0 & $0.0025 \pm 0.0001$ \\
\hline 10.0 & $0.0034 \pm 0.0001$ \\
\hline 20.0 & $0.0067 \pm 0.0001$ \\
\hline 30.0 & $0.0092 \pm 0.0003$ \\
\hline 50.0 & $0.0119 \pm 0.0005$ \\
\hline
\end{tabular}

Механизм окисления РС в присутствии перекиси водорода. Показано, что скорость реакции между двумя противоположно заряженными ионами уменьшается с увеличением диэлектрической проницаемости растворителя, а скорость реакции между ионом и нейтральной молекулой изменяется незначительно [11]. В водноорганических растворах при $\mathrm{pH}<8$ молекула $\mathrm{PC}$ заряжена положительно, следовательно, активные частицы окислителя имеют отрицательный заряд. $\mathrm{H}_{2} \mathrm{O}_{2}$ является слабой кислотой $\left(\mathrm{pK}_{\mathrm{a}}=11.75\right)$ [12], следовательно, в нейтральных растворах $\mathrm{PC}$ концентрация активной частицы окислителя $\mathrm{HO}_{2}{ }^{-}$низка. Ее участие в реакции окисления РС должно смещать равновесие диссоциации $\mathrm{H}_{2} \mathrm{O}_{2}$ в сторону образования $\mathrm{HO}_{2}^{-}$. Влияние ДМСО слабее, поскольку его диэлектрическая проницаемость выше, чем у $\mathrm{CH}_{3} \mathrm{CN}$ (48.50 и 35.94 соответственно [13]) и он менее значительно снижает эту характеристику водно-органического раствора. Кроме того, влияние ДМСО ослабляется его взаимодействием с $\mathrm{H}_{2} \mathrm{O}_{2}$ [14].

Согласно полученным результатам, основной продукт разложения РС при воздействии $\mathrm{H}_{2} \mathrm{O}_{2}$ имеет удерживание около 0.25 относительно РС (рис.2 а). Согласно USP36, анализ лекарственной формы РС «Раствор для приема внутрь» ведут в обращенно-фазовом режиме при рН ПФ около 7, при этом относительное время удерживания одного из основных продуктов разложения $\mathrm{PC}$ - рисперидона-цис-N-оксида - составляет 0.33 [2, стр. 5064-5065]. Другую лекарственную форму РС - таблетки жевательные согласно USP36 также анализируют в обращенно-фазовом режиме, но $\mathrm{pH}$ буферной составляющей ПФ в соответствующей методике равен 3.0; при этом время удерживания рисперидона-цис-N-оксида - около 1.70 относительно РС [2, стр. 5067-5068]. 


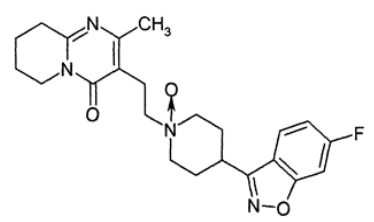

Такое резкое отличие хроматографических свойств рисперидона-цис-Nоксида по сравнению с РС, для которого наблюдается противоположная зависимость удерживания от $\mathrm{pH}$ ПФ, объясняется изменением третичного атома азота в процессе оксиления РС. Неподеленная электронная пара этого атома смещается к кислороду и уже не обеспечивает основные свойства молекулы, как у РС. Напротив, рисперидона-цис-N-оксид в обращенно-фазовой системе ведет себя, как кислота: протонизация атома кислорода группы $\mathrm{N} \rightarrow \mathrm{O}$ приводит к снижению полярности молекулы и, соответственно, к росту ее удерживания. Хроматограммы раствора РС после его деструкции в $3 \%$ растворе $\mathrm{H}_{2} \mathrm{O}_{2}$ при $\mathrm{pH}$ буферных составляющих 4.3 и 2.0 приведены на рис. 4 , а и б.
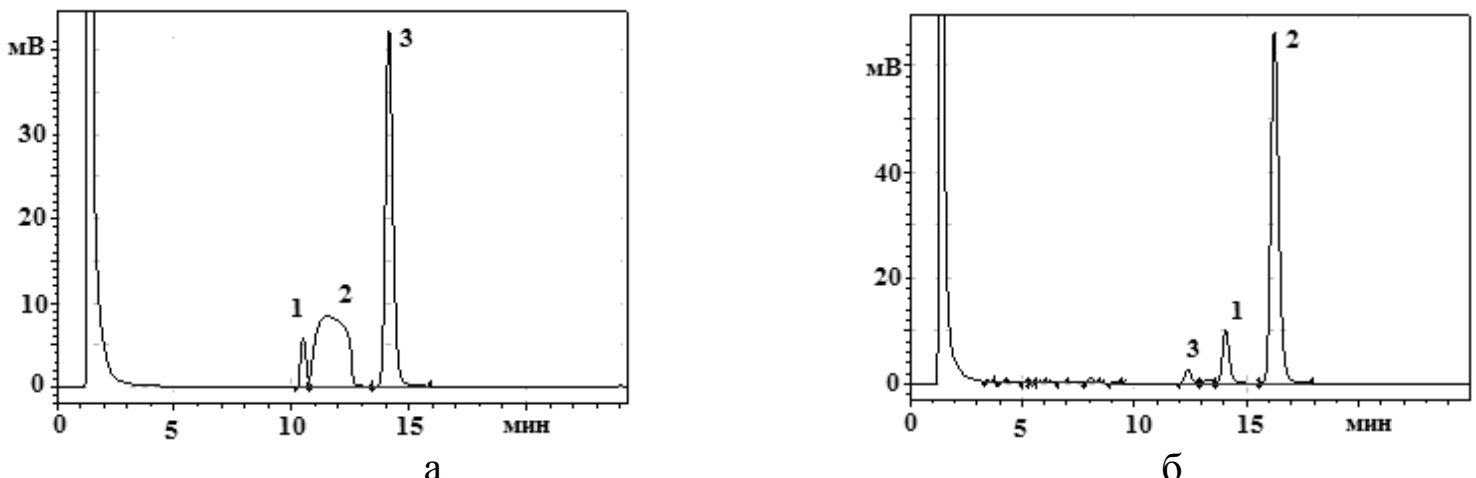

Рис. 4 Хроматограммы раствора РС (57.0 мг/см$\left.{ }^{3}\right)$ в смеси $(3: 7) \mathrm{CH}_{3} \mathrm{CN}-3 \%$ раствор $\mathrm{H}_{2} \mathrm{O}_{2}$. ПФ (23:77) $\mathrm{CH}_{3} \mathrm{CN}-0.5$ об. \% $\mathrm{HClO}_{4} \mathrm{pH} 4.3\left(\mathrm{NH}_{3}\right)$ (а) и (23:77) $\mathrm{CH}_{3} \mathrm{CN}-$ 0.5 об. \% $\mathrm{HClO}_{4} \mathrm{pH} 2.0\left(\mathrm{NH}_{3}\right)$ (б). Хроматограммы получены через 1 час (а) и через 28 часов (б) после приготовления раствора. 1 - неидентифицированный продукт разложения РС; 2 - рисперидон-цис-N-оксид; 3 - PC.

В первом случае, $\mathrm{pH}$ ПФ близок к значению $\mathrm{pK}$ продукта кислотной природы, и, как следствие, наблюдаются размывание его пика и неполное разделение с соседними пиками. При рН 2.0 буферной составляющей ПФ время удерживания РС 12.4 мин, время удерживания пика продукта разложения 16.2 мин (относительное время удерживания 1.31). Сопоставление литературных данных и полученных экспериментальных результатов позволяет предполагать, что этот продукт разложения РС рисперидон-цис-N-оксид, либо вещество, близкое ему по структуре. В пользу этого предположения свидетельствует и идентичность спектров поглощения РС и продукта разложения, детектируемых в режиме on line.

\section{Заключение}

Растворы РС, содержащие продукты разложения, целесообразно анализировать в градиентном режиме при изменении содержания ацетонитрила в ПФ от 20 до 30 об. \% в течение 20 мин, используя в качестве буферного компонента ПФ водный раствор хлорной кислоты (0.3-0.5 об. \%) с добавлением аммиака до $\mathrm{pH}$ 6.7-7.3. Такой подход позволяет полностью разделить анализируемые вещества. РС чувствителен к воздействию окислителей, и это необходимо учитывать при разработке состава, технологии лекарственных препаратов и методик анализа. Добавление в растворы РС 
ацетонитрила ускоряет разложение этого вещества при действии перекиси водорода с образованием рисперидона-цис-N-оксида. ДМСО более предпочтителен для пробоподготовки препаратов РС, поскольку его растворяющая способность выше, а скорость окисления РС ниже, чем в присутствии ацетонитрила. Для полного растворения вещества в растворы РС следует вводить, как минимум, 5 об. \% органического растворителя. Полученные результаты могут быть использованы при анализе пролонгированной лекарственной формы РС и разработке методик анализа других лекарственных средств.

\section{Список литературы}

1. Risperidone. Open Chemistry Database (PubChem). Compound Summary for CID 5073/U.S. National Library of Medicine. National Center for Biotechnology Information.

2. The United States Pharmacopoeia. The National Formulary (USP36-NF31) / 2013. Vol. 3. $1703 \mathrm{p}$.

3. Tomar R.S., Joseph T.J., Murthy A.S.R., Yadav D.V. et al. // J. Pharm. Biomed. Anal. 2004. Vol. 36. No 1. pp. 231-235.

4. Bhatt J., Subbaiah G., Singh S. // Rapid Commun. Mass Spectrom. 2006. Vol. 20. No 14. pp. 2109-2114.

5. Svirskis D., Travas-Sejdic J., Sanjay G. // J. Chromatogr. Sci. 2011. Vol. 49. No 10. pp. 780-785.

6. Барам Г.И., Рейхарт Д.В., Гольдберг Е.Д., Изотов Б.Н. и др. // Бюл. экспер. биол. и медииины. 2003. Т. 135. № 1. С. 75-79.

7. Азарова И.Н., Барам Г.И. // Сорбичонные и хроматографические прочессы. 2014. Т. 14. № 1. C. 65-74.

\section{References}

1. Risperidone. Open Chemistry Database (PubChem). Compound Summary for CID 5073/U.S. National Library of Medicine National Center for Biotechnology Information.

2. The United States Pharmacopoeia. The National Formulary (USP36-NF31) / 2013. Vol. 3.

3. Tomar R.S., Joseph T.J., Murthy A.S.R., Yadav D.V. et al., J. Pharm. Biomed. Anal., 2004, Vol. 36, No 1, pp. 231-235.

4. Bhatt J., Subbaiah G., Singh S., Rapid Commun. Mass Spectrom. 2006, Vol. 20, No 14, pp. 2109-2114.

5. Svirskis, D., Travas-Sejdic J., Sanjay G., J. Chromatogr. Sci., 2011, Vol. 49, No 10, pp. 780-785.

6. Baram G.I., Reichart D.V., Goldberg E.D., Izotov B.N. et al., Bull. eksper. Biol. and medicine, 2003, Vol. 135, No 1, pp. 75-79.
8. Jones A., LoBrutto R., Kazakevich Y. // J. Chromatogr. A. 2002. Vol. 964. No 1-2. pp. 179-187.

9. LoBrutto R., Jones A., Kazakevich Y. // J. Chromatogr. A. 2001. Vol. 913. No 1-2. pp. 189-196.

10. Kromidas S. Practical problem solving in HPLC. Weinheim: WILEY-VCH. 2004. 178 p..

11. Марк Г., Рехниц Г. Кинетика в аналитической химии. М. Мир. 1972. 368 с.

12. Hydrogen peroxide. Open Chemistry Database (PubChem). Compound Summary for CID 784. U.S. National Library of Medicine. National Center for Biotechnology Information.

13. Рудаков О.Б., Востров И.А., Федоров С.В., Филлипов А.А. и др. Спутник хроматографиста. Методы жидкостной хроматографии. Воронеж. Водолей. 2004. 528 с.

14. Химическая энциклопедия: В 5 т.: т. 2: Даффа-Меди / Редкол.: Кнунянц И.Л. (гл. ред.) и др. М.: Сов. энцикл. 1990. 671 с.

7. Azarova I.N., Baram G.I. Sorbtsionnye $i$ khromatograficheskie protsessy, 2014, Vol. 14, No 1, pp. 65-74.

8. Jones A., LoBrutto R., Kazakevich Y., $J$. Chromatogr. A, 2002, Vol. 964, No 1-2, pp. 179-187.

9. LoBrutto R., Jones A., Kazakevich Y., J. Chromatogr. A, 2001, Vol. 913, No 1-2, pp. 189-196.

10. Kromidas S., Practical problem solving in HPLC / Weinheim: WILEY-VCH. 2004, 178 p.

11. Mark G., Rechnitz G., Kinetika v analiticheskoj himii, M., Mir, 1972, 368 p.

12. Hydrogen peroxide. Open Chemistry Database (PubChem). Compound Summary for CID 784. U. S. National Library of Medicine. National Center for Biotechnology Information. 
13. Rudakov O.B., Vostrov I.A., Fedorov S.V., Filippov A.A. et al., Sputnik khromatografista. Metody zhidkostnoj khromatografii, Voronezh, Vodoley, Aquarius. 2004. 528 p. P. 295-296.

Голубицкий Григорий Борисович - старший научный сотрудник лаборатории систем доставки лекарственных средств, д.Х.н., ООО «Технология лекарств», Химки.

Владимирова Елена Валериевна - старший научный сотрудник лаборатории систем доставки лекарственных средств, к.Х.Н., ООО «Технология лекарств», Химки

Островский Константин Петрович - научный сотрудник лаборатории систем доставки лекарственных средств, ООО «Технология лекарств», Химки.

Гельперина Светлана Эммануиловна - начальник лаборатории систем доставки лекарственных средств, д.х.н., ООО «Технология лекарств», Химки.
14. Chemical encyclopedia: In 5 volumes, Vol. 2: Duffa-Medi/Redkol.: Knunyants, I. L. (ed.) and others. M.: Sov. encyclopedia, 1990, $671 \mathrm{p}$.

Golubitsky, Grigory B. - Senior researcher of the laboratory delivery systems of medicines, grand Ph.D Chemistry, LTD "Drugs Technology”, Khimki, e-mail: iirogirg@ @arod.ru

Vladimirova Elena V. - Senior researcher of the laboratory delivery systems of medicines, Ph.D. Chemistry, LTD "Drugs Technology", Khimki.

Ostrovsky Konstantin P. - Researcher of the laboratory of delivery systems of medicines, LTD “Drugs Technology”, Khimki.

Gelperina Svetlana E. - Head of the laboratory of delivery systems of medicines, grand Ph.D Chemistry, LTD "Drugs Technology”, Khimki. 